\title{
Proceedings of the Association of British Neurologists, Royal College of Physicians, London, 24-25 September 1992
}

MULTIPLE SCLEROSIS IN TWINS: THE BRITISH ISLES SURVEY

CJ Mumford, NW Wood, H Kellar-Wood, DAS Compston and the UK-MS Twin Study Group, University of Cambridge, UK

A total of 146 probands with multiple sclerosis (MS) who had a twin were identified in the United Kingdom and Ireland during a 27 month recruitment period. Direct personal interview and clinical examination of both twins was achieved in 106 cases (73\%).

In one case $(1 \%)$ the diagnosis of multiple sclerosis in the index case was rejected. In the remaining 105 pairs, 11 of 44 monozygotic pairs examined (25\%) were found to be clinically concordant for multiple sclerosis, and two of 61 dizygotic pairs (3\%) were clinically concordant. Zygosity was confirmed using mini-satellite probes.

These findings are similar to the results found in the large North American series of MS in twins $(n=70)$ and the Danish MS twins study $(n=60)$, and suggest a significant genetic component in the aetiology of the disease.

SPINAL CORD MAGNETIC RESONANCE IMAGING IN MULTIPLE SCLEROSIS USING MULTI-ARRAY COILS AND FAST SPIN ECHO

JW Thorpe, D Kidd, AJ Thompson, WI McDonald, DH Miller, Institute of Neurology, London, UK

Involvement of the spinal cord is responsible for much of the motor disability in multiple sclerosis (MS) but the cord has proved technically difficult and time-consuming to image by magnetic resonance due to its small size, poor signal to noise ratio, motion artefacts and limitations in surface coil design. Multi-array coils consist of a series of overlapping coils coupled electronically to give the coverage of a volume coil with the signal to noise advantages of a small surface coil. Fast Spin Echo (FSE) software affords a reduction in scanning time of up to 16 fold. The authors used multi-array coils and FSE at $1.5 \mathrm{~T}$ to obtain $\mathrm{T}_{2}$-weighted sagittal images of the whole spinal cord of 58 patients with MS in under 6 minutes. Lesions were detected in $31(74 \%)$. The mean number of lesions was 1.6 of which $74 \%$ were cervical and $26 \%$ thoracic. One lesion was seen in 40 healthy age matched controls.

In summary, this technique allows the ready identification of $M S$ lesions in the spinal cord and has considerable potential in the differential diagnosis of myelopathy.

MRI ACTIVTTY AND DISABILITY IN MULTIPLE SCLEROSIS: A TWO YEAR STUDY

D Kidd, AJ Thompson, DH Miller, AG Kermode, DG MacManus, WI McDonald, Institute of Neurology, London, UK

The aim of this study was to assess the relationship between MRI activity and the development of irreversible disability over time in multiple sclerosis (MS).

Forty four patients in the four clinical subgroups of MS (relapsing/remitting, benign, secondary progressive and primary progressive) were studied. Patients had clin- ical examinations and $T_{2}$-weighted $M R I$ brain scans at three monthly intervals for two years.

There were a total of 480 new lesions over the study period and 59 relapses. Most MRI activity was seen in the relapsing/remitting and secondary groups (a mean of 20 and 15 new lesions respectively) and least in the benign and primary progressive groups (mean of four and five new lesions respectively).

Patients who remained clinically stable had little MRI activity (mean $=5.6$ new lesions) while those who relapsed had greatest activity (15 in relapsing/remitting and benign groups and 18.5 in the secondary progressive group). Patients who progressed without relapse had the least MRI activity (mean $=4.3$ new lesions)

It is concluded that 1) clinical relapses are associated with more frequent brain MRI activity; 2) there is a relationship between brain MRI activity and change in disability over time in patients who relapse. The low brain MRI activity in progressive patients may be explained by relatively greater spinal cord involvement.

MULTIPLE SCLEROSIS AND THE T-CELL RECEPTOR $\beta$ LOCUS

NW Wood, CJ Mumford, MB Fraser, J Deans, DAS Compston, University of Cambridge, UK

Pedigree analysis and twin studies have implicated a genetic component in the aetiology of multiple sclerosis. The search for susceptibility genes has focused on candidates which regulate immune responses, especially polymorphisms encoded within the HLA system and T cell receptor loci. The method of identity by descent in 69 affected sibling pairs was used to investigate this locus in detail. Restriction fragment length polymorphisms have been used to assign parental haplotypes using at least three enzyme/probe combinations spanning the beta locus on chromosome 7q32-35 from the telomeric constant region ( $C$ beta) to the centrimerically placed $\mathrm{V}$ beta 1 . This combination has a high polymorphism information content (PIC) so that identity by state can be excluded even in those siblings whose parents were not available and in whom haplotypes could not directly be assigned. Identity by dexent analysis has demonstrated a bias in favour of haplotype sharing $(p<0.05)$ giving a maximum lod score of $1 \cdot 02$. Intrafamily association has identified a $\mathrm{V} \beta 8 / \mathrm{C} \beta$ haplotype conferring a relative risk of 3.9.

EFFECTS ON MOVEMENT OF SURGICAL INCISIONS INTO THE SPINAL CORD

PW Nathan, National Hospital for Neurology and Neurosurgery, London, UK

In 65 cases of anterolateral cordotomy, incisions into the spinal cord examined histologically were correlated with the disturbance of motility during life. Incisions dividing one anterior quadrant of the cord above the cervical enlargement caused no disturbance of motility. When this incision was bilateral below the cervical enlargement dividing the anterior half of the cord, surprisingly there was usually no disturbance of motility, although some patients felt that movements of the lower limbs required more effort.

One patient had the opposite kind of incisions in the mid-thoracic cord: one lateral corticospinal tract was completely divided, the other about $85 \%$ divided. At first there was total paraplegia; the patient was then able to sit; in four months he was walking, though with severe spastic paraparesis. Is this recovery of standing and walking due to the remaining $15 \%$ of lateral corticospinal fibres or was it due to other fibres from the brainstem? That it is due to fibres of the lateral corticospinal tract may be inferred from the existence of the locked-in syndrome, in which both corticospinal tracts are divided, and the patient is totally unable to move.

RECORDINGS FROM RAT DORSAL COLUMNS AND SPINAL ROOTS REVEAL A MECHANISM FOR ECTOPIC SPIKING

R Kapoor, KJ Smith, PA Felts, $M$ Davies, Institute of Neurology and Guy's Hospital, London, UK

It is not clear how ectopic firing and positive symptoms are generated in diseases affecting myelinated axons. Proposed mechanisms include crosstalk between adjacent nerve fibres and pathological excitatory currents arising in abnormal axonal membranes.

Extracellular microelectrode recordings were recently obtained from normal rat dorsal columns and spial roots in vivo and in vitro. The recorded potentials showed spontaneous cyclical shifts from a mean of $-4 \mathrm{mV}$ to a mean of $-27 \mathrm{mV}$ which dependent on the presence of potassium in the microelectrode solution. Negative potential shifts in vitro were blocked by the potassium channel blockers 4-AP (1-5 mM) and TEA (2-10 mM). Most significantly, ectopic firing was observed during negative shifts, particularly during their initial phases.

These findings suggest that in ectopic firing a build up of potassium ions in the periaxonal space may cause the normally outward internodal potassium currents to become inward, so depolarising adjacent nodes and generating spontaneous action potentials. Bursts of ectopic spikes resembling those described have also been observed in pathological situations including some peripheral nerve disorders and models of ischaemia and demyelination. It is possible that the underlying mechanisms may again involve paradoxical excitation of the axon by internodal potassium currents.

RESPIRATORY INVOLVEMENT AND ITS MANAGEMENT IN PRIMARY DISORDERS OF MUSCLF

RS Howard, CM Wiles, NP Hirsch, GT Spencer, The National Hospital for Neurology and Neurosurgery, and St Thomas' Hospital, London, UK

Eighty four patients with primary disorders of muscle were referred for assessment of respiratory insufficiency between 1975-91. The diagnoses were: "limb girdle syn- 
dromes" (18 patients), acid maltase deficiency (14), dystrophia myotonica (13), inflammatory disorders (10), congenital myopathies (9), rigid spine syndromes (5), dystrophies (Duchenne (6), fascioscapulohumoral (4), Becker (1)) and a miscellaneous group (4). Twenty eight patients subsequently died.

Presentation was often insidious with progressive nocturnal hypoventilation culminating in respiratory failure or arrest, recurrent respiratory tract infections or obstructive sleep apnoea. Respiratory symptoms developed relatively early in patients with acid maltase deficiency and inflammatory disorders but paralleled the development of limb weakness in limb girdle, myotonic and congenital syndromes.

Sixty six patients received respiratory support for a mean of 5.4 years. Various techniques of negative and positive pressure respiratory support were used. Tracheostomy was performed in 32 patients and used for domiciliary nocturnal ventilation in 22 .

Respiratory support is well tolerated, affords symptomatic relief and often prolongs survival in patients with primary disorders of muscle. The type of ventilatory support used depends on the clinical features and prognosis.

NEUROLOGICAL AND GENITOURINARY DYSFUNCTION IN MULTIPLE SYSTEM ATROPHY

R Beck, CD Betts, CJ Mathias, N Quinn, CJ Fowler. The National Hospital for Neurology and Neurosurgery, London, UK

The neurological and genitourinary symptoms in 51 patients with probable MSA (all with abnormal sphincter EMG) are described. Initial symptoms were genitourinary in $45 \%$, genitourinary and neurological in $23 \%$, neurological alone in $31 \%$ and laryngeal in $2 \%$. Impotence was the initial symptom in $67 \%$ of men and occurred 5.5 years before a diagnosis of MSA was made. At diagnosis the neurological features were protean: $75 \%$ had Parkinsonism, $76 \%$ autonomic failure, $43 \%$ pyramidal signs and $47 \%$ cerebellar dysfunction, $6 \%$ had all four. By this time $95 \%$ of the male patients had become impotent and all patients had significant bladder symptoms.

Urinary urgency affected $95 \%$ of men with $77 \%$ progressing to urge incontinence. Male patients also complained of difficulty initiating voiding and of voiding completely, causing chronic retention in $30 \%$. Symptomatology was similar in women except that $100 \%$ became incontinent, either stress $(43 \%)$ or urge $(47 \%) .43 \%$ of patients had undergone urological surgery prior to diagnosis, all without benefit.

A high post-micturition residual urine volume was a common finding (average $290 \mathrm{ml}$ ) and so effective treatment consisted of intermittent catheterisation. Anticholinergic medication was given for concomitant detrusor hyperreflexia and DDAVP spray for troublesome nocturia. $75 \%$ of patients regained continence.

ABNORMAL RNA PROCESSING ASSOCIATED WITH A NOVEL TRNA MUTATION IN MITOCHONDRIAL DNA LA Bindoff, N Howell, K Weber, J Poulton. University of Newcastle upon Tyne, UK, UTMB Galveston, Texas, USA, and University of Oxford, UK
Abnormalities of mitochondrial DNA (mtDNA) are increasingly being recognised in human disease. Point mutations in transfer RNA ( $t R N A$ ) encoded by mtDNA have been found in MERRF (myoclonus epilepsy with ragged-red fibres), MELAS (myopathy, encephalopathy, lactic acidosis and strokelike episodes) and a maternally inherited cardiomyopathy whilst mutations in proteincoding genes have been found in Leber's Optic Atrophy. Understanding of the mechanism whereby tRNA mutations produce their biochemical defect is incomplete although recent studies have shown that two are associated with a defect in intramitrochondrial protein translation.

A new mutation in tRNA ${ }^{\text {Leu(UUR) is des- }}$ cribed in a patient that died with a progressive myopathy. This mutation not only caused loss of tRNA ${ }^{\text {Leu }}$ but also affected processing of the adjacent mRNA encoding a subunit of complex 1 (ND1). The combined effect of lowered tRNA concentration and loss of ND1 product was accurately reflected in biochemical analysis suggesting both were involved in pathogenesis. In addition, studies have identified important tissue differences in the processing of mtDNA transcripts. These findings are the first clear linkage between abnormal mitochondrial RNA processing and disease and provide one possible mechanism whereby tRNA mutations generate their cellular effect.

EVIDENCE FOR AN AUTOANTIBODY MEDIATED MECHANISM IN ACQUIRED NEUROMYOTONIA

P Shillito, B Lang, J Newsom-Davis, B Bady, G Chauplannaz. Institute of Molecular Medicine, Oxford, and Hopital Neurologique, Lyon, France

Acquired neuromyotonia (NMT) is a syndrome of continuous muscle fibre activity due to hyperexcitability of peripheral nerves. The authors have reported improvement following plasma exchange in a single case and reduced sensitivity to d-tubocurarine at the neuromuscular junction of mice injected with the patient's plasma or IgG. The mechanism of this effect has now been investigated.

IgG was prepared from the plasma of three patients with clinical and EMG features of NMT, and from pooled control human plasma. Mice were killed after injection with $20 \mathrm{mg}$ IgG intraperitoneally for 10 days. Neuromuscular transmission was studied in vitro in the phrenic nerve-diaphragm preparation using conventional microelectrode techniques. Miniature endplate potential frequencies and amplitudes were normal. The quantal content of the end plate potential (m) was significantly increased for the NMT IgG injected mice (33.3 SEM +/- 1.4; $\mathrm{n}=70$ fibres, 12 mice; $\mathrm{p}=<0.001)$ compared with controls $25 \cdot 8+/-1 \cdot 5$; $n=48$, 8 ). When analysed individually, IgG from each of the three patients increased $m$ in injected mice, this being significant in two $(\mathrm{p}=<0.001$ and $\mathrm{p}=0.003)$.

These results, showing that NMT IgG increases the number of packages of neurotransmitter released by each nerve impulse $(m)$, would be consistent with a reduction in the number of functional potassium channels that normally regulate nerve excitability.

PLASMA EXCHANGE TREATMENT FOR GUILLAIN-BARRE SYNDROME

GDP Smith, RAC Hughes. Guy's Hospital, London, UK
A retrospective case notes analysis was made of 52 patients presenting to Guy's Hospital with Guillain-Barré syndrome (GBS) between August 1987 and September 1990. Twelve months after onset $61 \%$ had recovered completely, $35 \%$ were still significantly disabled and two patients (4\%) had died. A total of $48(92 \%)$ of the patients were treated with plasma exchange (PE). The frequency of morbidity related to PE was low and PE was not responsible for either of the deaths.

Outcome for all patients treated with PE between January 1985 and September 1990 was compared with 64 patients with GBS treated in 1983 and 1984 without PE. Time to walking without aid was significantly better (median 62.5 days compared with 86 days, $p=0.05$ ), as was the median duration of ventilation ( 16.5 compared with 36 days, $\mathrm{p}=0.001$ ).

Factors which had predicted an adverse outcome in previous studies; requirement for ventilation, age over 40 years, time to becoming bedbound less than four days and small distally evoked abductor pollicis brevis muscle action potential, were not significantly associated with a poor prognosis in this study. The features associated with persisting disability were time to improvement more than 21 days, preceding diarrhoea and older age.

DOUBLE-MASKED TRIAL OF INTRAVENOUS METHYLPREDNISOLONE IN GUILLAIN-BARRÉ SYNDROME

RAC Hughes for the Guillain-Barré Syndrome Steroid Trial Group, UMDS, Guy's Hospital, London, UK

To discover whether steroids are beneficial in Guillain-Barré syndrome (GBS), 242 patients were randomised in a multicentre trial to receive intravenous methylprednisolone (IVMP) $500 \mathrm{mg}$ daily for 5 days or placebo. Patients had to fulfil standard diagnostic criteria and be adult, so weak that they could not run, and within 15 days from the symptomatic onset of neuropathy. Plasma exchange was permitted but not mandatory. Disability grade was assessed on a scale ranging from $0=$ healthy to $6=$ dead at intervals until 48 weeks after randomisation. There was no significant difference in outcome between the 124 IVMP and the 118 placebo-treated patients on any outcome criteria, including the major outcome criterion, which was the change in disability grade four weeks after randomisation. The median time to walk unaided was 38 (95\% confidence limits [CL] 35-41) days in the IVMP group compared with 50 (95\% CL 20-80) days in the placebo group. There were five deaths and seven relapses in the IVMP group and two deaths and four relapses in the placebo group. After 48 weeks the mean (SD) disability grade was $1.33(1.56)$ in the IVMP group and $1.13(1.15)$ in the placebo group. The results do not suggest a beneficial role for IVMP in GBS.

ANTI-NERVE ANTIBODIES IN CHRONIC IDIOPATHIC DEMYELINATING POLY-RADICULONEUROPATHY NA Gregson, S Hall, RAC Hughes. UMDS Guy's Hospital, London, UK

The authors examined sera from patients with chronic idiopathic demyelinating polyradiculoneuropathy (CIDP) and other neurological diseases for antibodies against human peripheral nerve. Antibodies have 
been found against both protein and glycolipid antigens of human cauda equina. For screening by Western blotting, post-mortem cauda equina was homogenised in $0.32 \mathrm{M}$ sucrose. After centrifuging at $1000 \mathrm{rpm}$ for 10 minutes the supernatant was aliquoted and frozen. Samples containing $10 \mu \mathrm{g}$ of protein in SDS-page sample buffer were electrophoresed on 5-20\% acrylamide gradient gels, transferred to nitrocellulose and after blocking incubated overnight at $4^{\circ} \mathrm{C}$ with a 1/100 dilution of human serum. Bound immunoglobulin was detected with peroxidase conjugates. Sera of $7 / 23$ patients with CIDP contained IgG and/or IgM antibodies reactive with a minor $36-38 \mathrm{kDa}$ antigen found in extracts of human cauda equina but not in bovine CNS myeline. A similar antibody has been found in one of 19 OND controls. A sural nerve biopsy from one of the seropositive CIDP cases demonstrated active demyelination. The intraneural injection of 2 to $4 \mu \mathrm{l}$ of serum into the rat sciatic nerve from two of the patients with these antibodies produced vigorous primary demyelination, antibodies against this novel protein antigen may be important in demyelination.

MOLECULAR GENETIC STUDIES OF FAMILIAI AMYLOID POLYNEUROPATHY IN IRELAND AND THE UK

M Reilly, K Bhatia, MB Davis, H Staunton, PK Thomas, RHM King, MB Pepys, CH Hawkes, AE Harding. Institute of Neurology and Hammersmith Hospital London, UK and Beaumont Hospital, Dublin, Ireland

A cluster of patients with late onset amyloidosis presenting mainly with peripheral neuropathy and cardiac dysfunction has been reported in Donegal, North West Ireland. It was shown that two new patients from this region have a mutation of the transthyretin (TTR) gene which was initially described in a kindred with an Irish ancestor from the Appalachian region of the USA. Extended studies in Donegal demonstrate common ancestry among affected kindreds and pedigree analysis suggests reduced penetrance. Population studies in this region predict a frequency of asymptomatic gene carriers of $1 \cdot 3 \%$ (95\% confidence limits: $0-3 \cdot 1 \%$ ).

The TTR gene has also been analysed in patients from the UK who have amyloid neuropathy. One British patient (without a family history) had the met 30 (Portuguese) mutation, and another without German ancestry or affected relatives, had the tyr 77 (German) mutation. A third patient has a TTR mutation previously only described in one Japanese family (arg 50).

Genetic analysis of the TTR gene is a useful diagnostic procedure in patients with amyloid neuropathy regardless of family history. The demonstration of pathogenic mutations also has implications for genetic counselling.

UK NATIONAL AUDIT OF THE CARE OF COMMON NEUROLOGICAL DISORDERS

IMS Wilkinson on behalf of the Services Subcommittee of the Association of British Neurologists and the Research Unit of the Royal College of Physicians

Thirty four neurologists, from different regions of the UK, collected data on all new patients seen in a two week period in November 1991.

A total of 1620 patients were seen, of whom 1203 were suffering from the 16 most common neurological disorders. Of these 1203 patients: $61 \%$ were referred by general practitioners, $39 \%$ by hospital specialists; $50 \%$ were seen in regional neurological centres, $49 \%$ in district general hospitals, $1 \%$ on domiciliary visits; $80 \%$ were seen as outpatients, $20 \%$ as emergency admissions or ward referrals.

Of the 955 patients seen as outpatients, $61 \%$ were discharged after one attendance, $29 \%$ were requested to reattend, $10 \%$ were admitted. For the 184 patients admitted to a neurological bed, the average length of stay was 6.5 days.

For each diagnostic category, the following data are established (example data for epilepsy in brackets): number of patients seen (205); discharge, reattendance, admission figures for outpatients $(44 \%, 51 \%, 5 \%)$; mean length of admission, and total number of neurological bed days used (5.1 and 139 days); the use of diagnostic resources requesed by neurologist (EEG in $46 \%$, CT scan in $26 \%$ ); the use of treatment resources (drug therapy in $78 \%$ ); the number of patients changing diagnostic category as a result of investigation ( $2 \%)$.

COMPLICATIONS OF CEREBRAL ANGIOGRAPHY IN PATIENTS WITH SYMPTOMATIC CAROTID TERRTTORY ISCHAEMIA SCREENED BY CAROTID ULTRASOUND KN Davies, PRD Humphrey. Walton Hospital, Liverpool; UK

This is the first report of the complication rate for digital subtraction angiography in patients with carotid ischaemia who have been previously screened with carotid ultrasound so that those with normal or minimally diseased arteries do not proceed to angiog raphy. A prospective study of 200 consecutive patients was performed for local, systemic and neurological complications following carotid angiography defined as occurring within 72 hours of the procedure.

Complications occurred in 28 patients. There were six (3\%) local, two (1\%) systemic and $20(10 \%)$ neurological. The neurologica complications were transient in $10(5 \%)$ reversible in two (1\%) and permanent in six (3\%)). There were two deaths-in a patien who had an immediate stroke and died of a pulmonary embolism five days later and in a patient who had a severe stroke at 36 hours and died of convulsions and cerebral oedema. Of the other six permanent deficits, four were mild.

The incidence of angiographic complications was greater in patients with more than $90 \%$ stenosis and in those with persisting signs before angiography ( $<<0.001)$; it was also greater if performed by a senior registrar rather than a consultant ( $p<0.005)$.

During this same period 110 epileptic patients had undergone carotid angiography for Wada tests with three transient neurological events.

THE INTERNATIONAL STROKE TRIAL (PILOT) OF ANTITHROMBOTIC THERAPY IN ACUTE ISCHAEMIC STROKE: FOR WHOM MIGHT TREATMENT BE PARTICULARLY BENEFICIAL OR PARTICULARLY HAZARDOUS?

PAG Sandercock, CP Warlow, MS Dennis, RI Lindley. University of Edinburgh, UK
The International Stroke Trial (IST) is a large randomised controlled trial to determine whether or not, in patients with acute ischaemic stroke, immediate antithrombotic therapy (aspirin, subcutaneous heparin or the combination), started within 48 hours of onset reduces two week mortality by at least one sixth (for example, from $12 \%$ to $10 \%$ ) and reduces handicap in survivors. The aims of the pilot study are to: assess recruitment rates; exclude any very substantial hazards of therapy; determine whether a wide range of patients could be randomised. By 2 June 1992, 484 patients had been randomised in 18 centres (mean 2.5 patients/centre/month) The proportion of patients in ech category was: age $>65$ years $72 \%$; impaired conscious level $20 \%$; large MCA territory infarct $20 \%$; lacunar infarct $21 \%$; systolic $B P$ $>180 \mathrm{mmHg} 13 \%$. In all groups combined the two week mortality was $11.3 \%$ and the risk of non-fatal haemorrhagic transformation of the infarct was $8 \%$. The data monitoring committee have examined interim analyses and recommended that randomisation should continue. The main phase of the IST will begin in the UK in January 1993 and, if the expected 20000 patients are recruited, reliable evidence on the safety and efficacy of aspirin and heparin in the generality of patients with acute ischaemic stroke (and in several important specific categories of patient) will emerge.

THE RATE OF PROGRESSION OF CLINICAL AND SUBCLINICAL PARKINSON'S DISEASE GV Sawle, N Turjanski, DJ Brooks. MRC Cyclotron Unit, Hammersmith Hospital, London, UK

PET studies of patients undergoing foetal mesencephalic transplantation for Parkinson's disease have shown a progressive decline in $\left[{ }^{18} \mathrm{~F}\right]$ dopa uptake in un-operated striatal structures. The authors report data from five medically-treated and five transplanted patients scanned twice, 15 to 40 months apart. Standardised to a 24 month inter-scan interval, caudate and putamen uptake fell by $22 \%$ and $39 \%$ in medically treated patients, with $9 \%$ and $31 \%$ reductions in (un-operated) structures in transplanted patients. The greatest decline in the medically treated patients was in patients who were first scanned early in their clinical course.

Four controls have also been studied twice 35-46 months apart. Their data (standardised to the same inter-scan period) showed a fall of $0 \%$ for caudate and $5 \%$ for putamen in three cases, but $20 \%$ and $19 \%$ changes for the same structures in a fourth case. On the basis of his original scan, the fourth "control" had been identified as having possible subclinical Parkinson's disease. This further rapid decline supports that hypothesis, and suggests PET may be useful in determining the rate of progression of both clinical and subclinical Parkinson's disease.

LINKAGE STUDIES FOR CHROMOSOME 9Q32-34 IN BRITISH FAMILIES WITH IDIOPATHIC TORSION

TT Warner, MB Davis D Conway, NA Fletcher, F Ahmad, CD Marsden, AE Harding. Institute of Neurology, Queen Square, London, UK

Family studies of patients with generalised and segmental idiopathic torsion dystonia 
(ITD) in the UK suggest that $85 \%$ of cases are caused by an autosomal dominant gene(s) with $40 \%$ penetrance. We have analysed DNA polymorphisms mapping to chromosome 9q32-34 (the region containing the ITD locus in one non-Jewish and Jewish kindreds in the USA) in 26 small families with ITD, four Jewish and 22 non-Jewish For the microsatellite ASS locus, the combined lod score at $\theta=0.01$ was -3.8 . A large proportion of the negative score was derived from three non-Jewish families in which individual lod scores excluded a disease locus tightly linked to ASS. Statistical analysis showed significant heterogeneity. Exclusion of the three non-linked families gave maximum lod scores of $3 \cdot 2$ for ASS and 1.46 for $\mathrm{ABL}$ at $\theta=0.01$.

Affected members from three of the four Jewish kindreds had the ASS 12/ABL 4 haplotype, reported in linkage disequilibrium with ITD in Jewish families in the USA compared with $6 \%$ of Jewish controls $(p<0.001)$. No significant allelic association was seen in the index cases of the nonJewish families compared with non-Jewish controls. These data provide evidence for an OTD locus on chromosome 9q32-34 in both Jewish and non-Jewish kindreds in the UK, but also suggest genetic heterogeneity.

NEUROPATHIC AND ESSENTIAL TREMORS ARE BOTH ASSOCIATED WITH ABNORMALLY INCREASED ACTIVITY IN CEREBELIAR PATHWAYS

IH Jenkins, PG Bain, JG Colebatch, PD Thompson, RSJ Frackowiak, LJ Findlay, PK Thomas, CD Marsden, DJ Brooks. MRC Cyclotron Unit, Hammersmith Hospital, and MRC Human Movement and Balance Unit, London, UK

It has been proposed that essential tremor (ET) arises from central pathways; animal models implicate olivo-cerebellar connections. It is not known whether neuropathic tremor (NT) is central or peripheral in origin.

Regional cerebral blood flow (rCBF) was measured for $11 \mathrm{ET}$ and seven NT patients, and eight age matched controls using $\mathrm{C}^{15} \mathrm{O}$ PET. rCBF measurements were made unde three conditions in each group; rest without tremor, involuntary postural tremor on arm extension, and passive wrist oscillation at tremor frequency (ET and NT); rest, arm extension, and voluntary wrist oscillation mimicking postural tremor (controls). Resting cerebellar rCBF was significantly increased globally in ET and NT patients compared with controls $(p<0.01)$. Involun tary postural tremor was associated with further bilateral cerebellar activation in ET and NT, and also contralateral striatal and sensorimotor cortex activation. Additional activation of the contralateral thalamus was found in ET. Mimicked tremor and arm extension in control subjects produced only ipsilateral, not global cerebellar activation.

It was concluded that ET and NT are associated with increased bilateral cerebellar activity at rest and during involuntary central tremor generator in both these conditions, probably involving olivo-cerebellar connections.

GENETIC MUTATION IN CEREBRAL PALSY

NA Fletcher, J Foley. St Bartholomew's Hospital, and the Cheyne Centre for Spastic Children, London, UK
The cause of cerebral palsy is largely unknown; birth asphyxia accounts only for minority of cases. The parental ages at birth and order of birth in 251 cases of CP were studied to examine the hypothesis that some cases of CP arise because of fresh genetic mutation. Increased paternal age is associated with new mutation and has been observed in several other disorders which arise by this genetic mechanism.

Among the cases, parental ages and order of birth were not significantly different from those in the UK population in 50 cases of spastic quadriplegia and 75 cases of spastic diplegia. In athetoid CP (77 cases) paterna age was significantly increased $(+2 \cdot 16$ years, $P<0.02)$ and this was still the case afte allowance for increased birth order $(+1.78$ years, $P<0.02)$. In congenital hemiplegia paternal age was also increased $(+2 \cdot 6$ years, $P<0.02)$ but just so after allowance for birth order $(+2 \cdot 17$ years, $P<0.05)$.

These data suggest that some cases of athetoid CP arise due to new dominan genetic mutation. This is less certain in congenital hemiplegia. Such a genetic basi of athetoid CP may not be immediately apparent because of the low biological fitnes of this disorder.

VITAMIN B12 METABOLISM IN MULTIPLE SCLEROSIS T Bottiglieri, M Laundy, RF Crellin, SG Kirker, EH Reynolds. King's College Hospital, London, UK

The authors have recently reported 10 patients with multiple sclerosis (MS) associated with unusual vitamin B12 deficiency. We have therefore studied vitamin B12 metabolism in 29 consecutive cases of MS 17 neurological controls, and 31 norma subjects. Patients with MS had significantly lower serum vitamin B12 and significantly higher unsaturated R-binder capacity than neurological and normal controls, and were significantly macrocytic compared to norma controls. Nine patients with MS had serum vitamin B12 levels less than $200 \mathrm{pg} / \mathrm{ml}$ and in the absence of anaemia this subgroup was significantly macrocytic and had significantly lower red cells folate levels than neurological and normal controls. Patients with low serum vitamin B12 had raised total plasma homocysteine levels, indicating functional deficiency of vitamin B12. Nine patients with MS had raised plasma unsaturated $R$-binde capacity, including three patients with very high values. There is a significant association between MS and disturbed vitamin B12 metabolism. Vitamin B12 deficiency should always be looked for in patients with MS.

RISK FACTORS FOR MOTOR NEURON DISEASE: A POPULATION BASED CASE CONTROL STUDY AM Chancellor, H Fraser, JM Slattery, CP Warlow. University of Edinburgh, UK

One framework for research into the aetiology of motor neuron disease (MND) is to consider the potential interaction of ageing with environmental factors which may be remote from the onset of clinical disease. A case-control study has been carried out of incident patients in Scotland identified using the Scottish Motor Neuron Disease Register-a prospective collaborative epidemiological study of MND. A standardised questionnaire was administered to 103 patients and the same number of community controls matched on a one to one basis using the general practitioner's (GP) age and sex register. Recall bias was minimised by using GP notes to verify the subject's report. There was an overall lifetime excess of fractures in patients, Odds ratio $(\mathrm{OR})=1.3(95 \% \mathrm{CI}$ $0.7-2.5)$ and this was highest in the five years before symptom onset, $\mathrm{OR}=15$ (3.3-654)

The OR for a manual occupation was $2 \cdot 6$ $(1 \cdot 1-6 \cdot 3)$ and both exposure to lead OR $=5.3(1.5-28)$ and solvents/chemicals $\mathrm{OR}=3.8(1.5-11)$ were significantly more common in patients. No consistent association was found between MND and factors reflecting socioeconomic deprivation in childhood; childhood infections; social class or a number of medical illnesses. The limited extent of any single association suggests that the aetiology of MND may be multifactorial.

DISCONTINUATION OF CLONAZEPAM IN PATIENTS WITH ACTIVE EPILEPSY

J Chataway, A Fowler, PJ Thompson, IS Duncan. Institute of Neurology, London and Chalfont Centre for Epilepsy, Buckinghamshire, UK

The effects of withdrawal of clonazepam (CZP) at a rate of $1 \mathrm{mg} /$ week are reported in 23 patients with chronic active epilepsy. Seventeen (74\%) discontinued CZP successfully. No clinical features were identified that were associated with an increased risk of failure of CZP withdrawal. In the six patients in whom seizures increased, reintroduction of CZP rapidly resulted in control.

No significant change in cognitive function, mood or behaviour was noted in the patients who discontinued CZP. It is concluded that a rate of reduction of CZP of $1 \mathrm{mg} /$ week is a safe and reasonable figure.

OLFACTORY DYSFUNCTION IN PARKINSON'S DISEASE: NEW EVIDENCE FROM IDENTIFICATION TESTS, EVOKED POTENTIALS AND OLFACTORY BULB PATHOLOGY

CH Hawkes, B Shephard, SE Daniel. Ipswich Hospital, and Institute of Neurology, London, UK

Olfaction is profoundly disturbed in Parkinson's Disease (PD) and it has been suggested this may have a bearing on its cause. We have analysed the defect in detail as follows: three techniques were implemented a) University of Pennsylvania Smell Identification Test (UPSIT) in 96 patients and 96 controls; b) olfactory evoked responses (OEP) to 20 vppm hydrogen sulphide in 37 patients and 25 controls; c) Histological examination of olfactory bulbs from eight PD cases and eight age-matched controls. Sections were stained with haematoxylin-eosin and immunocytochemistry for ubiquitin and were examined "blind" to clinical diagnosis.

UPSIT scores were highly abnormal with over $80 \%$ scoring below the $95 \%$ confidence limit ( $p<0.0001)$. Certain odours presented more difficulty for patients than age matched controls, that is, pizza, lemon, rose, wintergreen and cloves, inferring a specific anosmia. In 15 patients the OEP was absent and in the other 22 there was significant delay. ( $p<0.0022$-sample $T$ test). In $50 \%$ of those with a normal UPSIT score there was delay on OEP. All PD olfactory bulbs contained Lewy bodies and were thus easily distinguished from controls.

Severe abnormalities of smell function were found in all three tests in almost every 
patient. It was demonstrated for the first time that the identification defect is selective and that diagnostic abnormalities may be found consistently in the olfactory bulbs.

NEUROPATHY IN MADELUNG'S SYNDROME: TWO CASES WITH UNUSUAL FEATURES

CH Hawkes, PK Thomas. Ipswich Hospital and Institute of Neurology, London, UK

Madelung's syndrome (1888) is characterised by multiple symmetrical lipomata present mainly over the trunk and in a "ruff" around the neck. It may be associated with distal sensiromotor neuropathy. Although the neuropathy was once attributed to alcoholism this view has now been discounted. The pathological basis of the neuropathy is an axonopathy. The disorder is possible of autosomal recessive inheritance. The authors report two cases of Madelung's disease with unusual features. In one the calf muscles were strikingly hypertrophied and muscle cramps were a prominent symptom. Her parents were consanguinous suggesting autosomal recessive inheritance. In the other patient, the neuropathy was accompanied by progressive limb ataxia despite only slight loss of joint position sense.

Because of the rarity of this syndrome there was considerable delay in reaching a diagnosis, but once seen, the phenotype is not readily forgotten.

UNILATERAL MEDULLARY LESIONS CAN DECPRESS $\mathrm{CO}_{2}$ SENSITIVITY AND MINIMALLY AFFECT RESPIRATION IN HUMAN SUBJECTS

P Heywood, S Moosavi, MJ Morrell, RJM Lane, J Stevens, A Guz. Charing Cross Hospital, and St Mary's Hospital, London, UK

Unilateral lesions in the medulla may cause Ondine's Curse. The authors studied patients with unilateral medullary lesions and found sleep apnoea to be common. When awake, do patients with lower brainstem lesions a) have normal $\mathrm{CO}_{2}$ sensitivity; b) breathe normally at rest? Lesions in 14 patients were located clinically and by MRI. All had $\mathrm{CO}_{2}$ sensitivity measured by rebreathing. During resting wakefulness $R R$, $V, T, T_{e}, V_{E}, \mathrm{PetCo}_{2}$ and $\mathrm{SaO}_{2}$ were recorded. Of the 14 patients, six had diminished $\mathrm{CO}_{2}$ sensitivity (slope: $\bar{x} \pm \mathrm{SD}=0.66 \pm 0.325 \mathrm{Lmin}^{-1} \mathrm{mmHg}^{-1}$ ), eight had normal $\mathrm{CO}_{2}$ sensitivity (slope: $=2 \cdot 80 \pm 0.51$ ) (normal values; slope: $=3.5 \pm 1 \cdot 3$ ). Lesions associated with diminished $\mathrm{CO}_{2}$ sensitivity were all in the rostro-lateral medulla. Although one of the patients with normal sensitivity also had a lesion in that area, lesions in the others were in the medial or caudal medulla, lower pons or cerebellum. Breathing during resting wakefulness in the group with low $\mathrm{CO}_{2}$ sensitivity was significantly slower $(\bar{x} R R \quad 12 \cdot 5$ v. $16.1 ; \mathrm{p}<0.03)$ due to prolonged $T_{0}\left(\bar{x} T_{\text {e }}\right.$ 4.08 v. $2.55 ; \mathrm{p}<0.03)$; no other breathing parameters differed significantly. Damage to the rostro-lateral part of the medulla oblongata, even when unilateral is likely to impair $\mathrm{CO}_{2}$ sensitivity: with minimal effect on breathing awake at rest; contrasting with the respiratory problems seen during sleep.

SLEEP APNOEA IN PATIENTS WITH CAUDAL

BRAINSTEM LESIONS

P Heywood, MJ Morrell, RJM Lane, J

\begin{tabular}{llll}
\hline Patient & Lesion & Type of apnoea & Min Nrem $\mathrm{SaO}_{2} \%$ \\
\hline 36 female & Caudal pons, MS & - & 94 \\
26 male & Caudal pons, MS & $\overline{\mathrm{O}}$ & 93 \\
64 female & Caudal pons \& rostral medulla, CVA & - & 89 \\
42 male & Rostro-lateral medulla, CVA & $\mathrm{M}$ & 94 \\
59 male & Rostro-lateral medulla, CVA & $\mathrm{M}$ and O & 80 \\
69 male & Rostro-lateral medulla, CVA & $\mathrm{O}$ & 85 \\
47 male & Rostro-lateral medulla \& cerebellum, CVA & C & 94 \\
38 male & Rostro-lateral medulla, CVA & C and O & 94 \\
44 male & Rostro-lateral medulla, CVA & O & 92 \\
52 male & Rostro-lateral medulla, CVA & O and M & 85 \\
61 male & Medial medulla, CVA & - & 93 \\
58 male & Pyramid \& ventral pons, CVA & - & 94 \\
68 male & Cervicomedullary, CVA & &
\end{tabular}

Stevens, A Guz, Charing Cross Hospital, and St Mary's Hospital, London, UK

Medullary respiratory centres (MRC) are responsible for "automatic" respiratory control. Attempting to better locate the human MRC the authors studied respiratory control awake and asleep in patients with medullary disease; results of cardio-respiratory polysomnography in 14 patients with lower brainstem lesions are reported. Location of the lesions was ascertained clinically and by MRI.

It was proposed that the pathophysiology was: in the $\mathbf{5 8}$ year old male, tongue paresis causing obstruction, in the other cases of obstruction, incoordination of MRC output to respiratory muscles and muscles maintaining upper airway patency, and in cases of central apnoea MRC dysfunction. Where desaturation is demonstrated CPAP should be considered to avoid dangerous nocturnal desaturation in patients whose vascular supply is already precarious.

A CASE OF OBSTRUCTIVE SLEEP APNOEA AFTER MEDIAL MEDULLARY INFARCTION

P Heywood, MJ Morrell, RJM Lane, J Stevens, A Guz. Charing Cross Hospital and St Mary's Hospital, London, UK

Obstructive sleep apnoea has been reported in various medullary disorders, but not hitherto in medial medullary syndrome. Seven years after a stroke a 59 year old man complained of excessive daytime somnolence and extreme lethargy; his wife recounted loud snoring with apnoiec pauses during sleep; these symptoms began after his stroke. Contralateral pyramidal weakness and ipsilateral tongue weakness were present, although dorsal column sensation was preserved. MRI confirmed the location of the infarction. His body mass index was 28 . Overnight polysomnography demonstrated severe obstructive sleep apnoea: a sleeping pattern disturbed by frequent electro-cortical arousals (60/hour) occurring at end of episodes of upper airway obstruction, during all stages of sleep. Obstructions resulted in desaturations $\left(\mathrm{SaO}_{2}\right.$ range in NREM $98 \%-82 \%$; awake $98 \%-92 \%$ ). CPAP (9 cm $\mathrm{H}_{2} \mathrm{O}$ ) was sufficient to abolish all apnoeas, desaturations and snoring. Polycythaemia ( $\mathrm{Hb} 18.7 \mathrm{~g} / \mathrm{dl}$ ) secondary to nocturnal hypoxaemia necessitated venesection before the introduction of long-term CPAP alleviated the problem. The pathophysiology in this case was a XII nerve paresis allowing the tongue to prolapse into the pharynx during sleep and obstruct the upper airway. The case highlights the need for awareness of upper airway problems in management of patients with tongue and pharyngeal problems.
RESPIRATORY ABNORMALITIES DUE TO ATLANTOAXIAL DISLOCATION

RS Howard, NP Hirsch, F Henderson, AH Crockard. The National Hospital for Neurology and Neurosurgery, London, UK

We investigated 11 patients with atlantoaxial subluxation due to rheumatoid disease for evidence of respiratory impairment. There were nine females and one male with a mean age of 64.7 years and a mean duration of rheumatoid disease of 22 years. All the patients were severely disabled by their underlying disease. At presentation eight patients had neck pain, seven limb weakness, six impaired mobility, three sensory symptoms and two bulbar weakness. Only one patient had symptoms suggestive of nocturnal hypoventilation. Three patients had significant myelopathy, two kyphoscoliosis and one bulbar weakness. CT myelography (10) and/or MRI scan (3) showed atlantoaxia dislocation in all patients with medullary compression in four and severe cervical cord compression in three.

Nocturnal oximetry revealed severe hypoventilation in the three patients with medullary compression. All the patients with cord compression had mild-moderate hypoventilation and there were no significant respiratory abnormalities in the absence of cord compression. In one patient studied following transoral odontoidectomy there was complete resolution of severe hypoventilation.

Nocturnal respiratory abnormalities occur in patients with medullary and spinal cord compression due to atlantoaxial subluxation. Whilst respiratory insufficiency in rheumatoid disease is multifactorial, significant compression of the neuraxis apparently contributes to both obstructive sleep apnoea and impaired central respiratory control.

THE USE OF ELECTRICAL IMPEDANCE TOMOGRAPHY TO MEASURE PHARYNGEAL TRANSIT TIME IN NEUROGENIC DYSPHAGI

TAT Hughes, P Liu, H Griffiths, CM Wiles. University Hospital of Wales, Cardiff, UK

In neurogenic dysphagia swallowing speed is usually slow commonly due to delay in the oral and/or pharyngeal phases. The authors have recently shown that it is possible to image a single bolus of liquid being swallowed using Electrical Impedance Tomography (EIT) and that a parameter of pharyngeal transit time can be computed: $362.9 \pm 106.9 \mathrm{~ms}$, mean (2 SD). The transit time was independent of bolus volume in the range $10-40 \mathrm{mls}$.

Two patients with brain stem vascular lesions and one with motor neuron disease have been studied. The EIT pharyngeal transit time for all three was compared with 
values obtained from a timed test of swallowing which yielded volume per swallow, volume per second and time per swallow. The results suggest that the pharyngeal phase is prolonged in some patients with dysphagia who have a slow swallowing speed associated with a reduced volume per swallow.

The technique offers the potential for non invasively identifying and monitoring prolonged pharyngeal clearance in neurogenic dysphagia.

MOTOR NEURON DISEASE: PREVALENCE AND DISABILITY IN SOUTH GLAMORGAN

CM James, PS Harper, CM Wiles. University Hospital of Wales, Cardiff, UK

Motor neuron disease (MND) although relatively rare in the general population, causes considerable disability in affected individuals. The authors estimated the disability caused by MND in South Glamorgan by measuring the prevalence of ND in this population through the use of an inpatient index and through contacting all general practitioners in the area. Subsequently identified individuals were clinically reassessed and classified using standard criteria. Disability was assessed using a modified Barthel Index and a mobility score. Each subject also had a quantitative assessment of swallowing.

A total of 25 patients previously diagnosed as having MND, resident in South Glamorgan on a specified day were identified, giving a prevalence of $4 \cdot 6 / 100,000$; none of these cases were familial. A total of 19 agreed to be assessed; of these 17 fulfilled criteria for either definite, probable, possible, or suspected ALS. The range of disease duration was $0.8-7.5$ years (median-4.0 years) $10 / 19(53 \%)$ scored 15 or less on the Barthel Index and $14 / 19(74 \%)$ had some degree of mobility impairment, $6 / 19$ (32\%) were wheelchair dependent. Swallowing speed was reduced $(<10 \mathrm{ml} / \mathrm{s})$ in $17 / 19(89 \%)$, but only 13 of these patients had clinical evidence of either bulbar or pseudobulbar weakness.

THE ROLE OF A NEUROREHABILITATION UNIT-ONE YEAR'S EXPERIENCE

D Kidd, RS Howard, J Ball, J Baldry, G Stewart, D Rossiter, J Hustler, N Fox, H Crimlisk, N Losseff, AJ Thompson. The National Hospital for Neurology and Neurosurgery, London, UK

As part of the continuing evaluation of neurological rehabilitation, one hundred and thirty four patients admitted to a neurorehabilitation unit over a twelve month period were studied. There were 53 males and 81 females with a mean duration of admission of $20 \cdot 2$ days. Diagnoses included multiple sclerosis (61 patients) cerebrovascular disease (25), hemisphere tumours (7), neuromuscular disease (13), other spinal cord syndromes (20) and a miscellaneous group (8).

The patients were treated by a multidisciplinary team and were clinically assessed and rated on admission and discharge using Barthel index (BI), environmental status scale (ESS) and a timed 10 metre walk.

Improvement in Barthel index was greater in patients with acute stroke and inflammatory neuropathies but also occurred in hereditary neuromuscular disorders and stable multiple sclerosis (MS). In the non MS group there was a close correlation between changes in BI and ESS. Time walking did not correlate with other measures of functional change in the whole group.

Seventy five per cent of patients showed functional improvement following neurorehabilitation. The greatest benefit was seen in association with some degreee of neurological recovery but it was possible to improve disability in patients with stable or deteriorating impairment.

REHABILITATION IN MULTIPLE SCLEROSIS-A

PRELIMINARY STUDY

D Kidd, RS Howard, N Losseff, AJ Thompson. The National Hospital for Neurology and Neurosurgery, London, UK

The place of rehabilitation in the management of multiple sclerosis (MS) remains uncertain. To address this question 61 patients with MS were studied who were admitted over a twelve month period for rehabilitation. There were 38 females and 23 males (mean disease duration, $12 \cdot 1$ years (1-37)). Eleven patients were recovering from a recent relapse and fifty were stable or had deteriorating neurological impairment.

The patients were clinically assessed and rated on admission and discharge using Kurtzke DSS. Barthel index and Environmental status scale (ESS) and where treated by a multidisciplinary team. The mean duration of admission was $14 \cdot 8$ days (1-59).

The mean DSS on admission was 6.5 (4-9), this remained unchanged in 46 and reduced by one or more points in 15 Reduction of DSS was either associated with remission or represented improvement in transfers. The mean Barthel on admission was $13.7(0-20)$ and improved by $>2$ in $65 \%$ of patients by discharge. The Barthel index was particularly sensitive to functional improvement in the moderate to severely disabled. The scale confirmed functional improvement of $88 \%$ of patients recovering from a recent relapse and also $62 \%$ with stable or deteriorating neurological impairment.

These results suggest a useful role for neurorehabilitation in the management of patients with MS including those with progressive disease.

ABNORMAL VISUOMOTOR PERFORMANCE IN A PATIENT WITH CALLOSAL AGENESIS

MC Lawden, P Brent, TC Crawford, CK Kennard, Charing Cross Hospital, London, UK

A 27 year old epileptic, with total agenesis of the corpus callosum on MRI, allowed investigation of the role of inter-hemispheric connections in visuomotor processing. Abnormal visual processing was demonstrated using apparent motion. When two lights 16 degrees apart on opposite sides of the midline were illuminated sequentially, a time delay of $200 \mathrm{~ms}$ was needed by the patient for optimal motion perception, while a control required only $100 \mathrm{~ms}$. If both lights lay in the patient's same hemifield, optimal delay fell to $100 \mathrm{~ms}$. In visuomotor function, reflexive saccade latencies to a novel stimulus were normal (mean $232 \mathrm{~ms}$ ), though amplitudes were hypometric (gain 0.68 ). In the anti-saccade task subjects respond to illumination of a peripheral target by making a saccade to the mirror-image position in the opposite hemifield. Seventy three per cent of the patient's initial anti-saccades were made erroneously to the triggering stimulus rather than into the opposite hemifield; the mean error rate of 30 controls was $21 \%$. This was not because the patient failed to comprehend the task, as $97 \%$ of his errors were immediately corrected. One possible explanation is that the inhibitory pathway by which inappropriate reflexive saccades are suppressed may involve inter-hemispheric connections, perhaps from the stimulated visual cortex to the contralateral frontal lobe.

TIMING OF MENTALLY REPRESENTED ACTIONS IN PARKINSON'S DISEASE

AP Moore, J Maraganore, CD Marsden. Institute of Neurology, London, UK

The authors investigated the idea that patients with Parkinson's disease (PD), who have perturbed motor executive mechanisms, may have relative sparing of mechanisms serving mental imagery of the same motor tasks.

The performance of 21 patients with idiopathic PD and slowed walking was compared with that of 22 healthy age-matched controls in the following task. Subjects timed themselves with a stopwatch as they walked $5 \mathrm{~m}$ or $10 \mathrm{~m}$ at a comfortable pace (Actual Walking Time or AWT), and again while imagining the same walk but actually standing still with eyes shut (Mental Walking Time, MWT). For each subject an average time was found for 10 walks in each condition. The sequence of actual and mental walking and the distance walked varied randomly.

Normal subjects could match AWT and MWT but in PD patients MWT was on average $10 \%$ faster than AWT, ANOVA showed that at both $5 \mathrm{~m}$ and $10 \mathrm{~m}$ this difference between the groups was significant (F-ratio $>6, p<0.02$ ). This could not be explained by age or gender differences, or by the slower AWT in PD.

This supports the theory that mental imagery and motor executive mechanisms are separate and can be differentially affected in PD.

CLINICAL AND ELECTROGRAPHIC FEATURES OF OCCIPITAL LOBE SEIZURES

S Sveinbjornsdóttir, JS Duncan, S Smith. The National Hospitals for Neurology and Neurosurgery, London and Chalfont St Peter, UK

A three year retrospective and a two year prospective study at The National Hospitals for Neurology and Neurosurgery revealed 27 patients with partial epilepsy likely to be of occipital origin. Elementary visual hallucinations occurred at the onset of seizures in 15 patients, loss of vision in three and ocular sensation in four. Subsequent manifestations depended on seizure spread. Two patients, who had a structural lesion in the occipital lobe did not have visual/ocular symptoms during their seizures, which mimicked temporal lobe epilepsy. Neuroimaging (CT/ MRI) was abnormal in 14 cases. Four patients had a space occupying lesion and three had vascular lesions. Other aetiologies included Rasmussen's encephalitis, mitochondrial cytopathy, perinatal and traumatic brain lesions and history of encephalitis. The epilepsy was apparently cryptogenic in 12 cases and one patient was considered to have benign occipital epilepsy of childhood. Three patients presented with migraine like symptoms, later evolving to overt seizures. Uni- 
lateral occipital, posterior temporal or posterior quadrant interictal epileptiform or slow activity was found in nine cases, corresponding to a structural lesion in six. Intermittent bioccipital spikes or sharp activity, sometimes involving a large posterior zone, were present in six cases and the interictal EEG was normal in four.

THE EFFECT OF IMMOBILISATION ON JOINT POSITION SENSATION

A Swinkels, CD Ward, University of Southampton, UK

Some theorists of motor skill acquisition imply that joint position sensation (JPS) can be improved through practice. Conversely, motor imbalance following periods of bedrest is sometimes attributed to proprioceptive deconditioning. We investigated the effect on JPS of chronic immobilisation of the knee. Subjects were orthopaedic patients including 10 in whom one knee had been immobilised in a long-leg plaster, and eight on strict bedrest, for two or more weeks; there were 30 matched controls. JPS threshold was determined by passive movement of the knee at $0.5 \mathrm{deg} / \mathrm{s}$. In three different reproduction tests the subject judged whether a knee movement had reproduced the same angle as the opposite knee. The test knee and the reference knee were moved either actively or passively. Exteroceptive and auditory cues were eliminated or minimised. There was no significant difference in JPS between normal and immobilised knees. In all groups mean JPS threshold was 1-2 degrees; there were frequent illusions of movement. Reproduction errors were 3.5 degrees or less; errors were higher for the active/passive than for the passive/passive and active/active conditions. The results provide no evidence of plasticity in the proprioceptive system.

SLEEP PROBLEMS IN MULTIPLE SCLEROSIS N Tachibana, RS Howard, NP Hirsch, DH Miller, WI McDonald, D Fish. The National Hospital for Neurology and Neurosurgery, London, UK

Twenty eight consecutive patients with multiple sclerosis (MS) were clinically evaluated to determine the presence of sleep related disorders. There were 12 males and 16 females aged between 22 and 67 with disability ranging between 1.5 and 8.5 on Kurtzke EDSS.

Fifteen patients $(54 \%)$ reported sleep related problems. These included difficulties initiating sleep and/or frequent awakenings due to spasms or discomfort in the legs (six patients), difficulty in initiating or maintaining sleep (3), habitual snoring (4) and involuntary movements during sleep (2).

All night oximetry was performed and the tracings analysed for the number of dips in oxygen saturation $\left(\mathrm{SaO}_{2}\right)$ of more than $4 \%$. Three patients showed significant sleep related oxygen desaturation $(>5$ dips of $>4 \% \quad \mathrm{SaO}_{2}$ /hour). Subsequent polysomnography performed in two of these confirmed the presence of sleep apnoea.

Specific MRI analysis of brain stem regions showed abnormalities in 20/22 cases. The three patients showing nocturnal oxygen desaturation had MRI brain stem lesions, but their locations were variable and their general appearance not different from that seen in the 17 without sleep apnoea.

Sleep disturbance in MS may be due to nocturnal respiratory insufficiency, but is much more often due to other factors including leg spasms, pain, immobility, or medication.

RECOVERY OF THE HYPOTHALAMIC-PITUITARYADRENAL (HPA) AXIS FROM SUPPRESSION BY SHORT TERM HIGH DOSE INTRAVENOUS PREDNISOLONE IN PATIENTS WITH MULTIPLE SCLEROSIS

G Wenning, H Wiethoelter, G Schnauder, PH Mueller, S Kanduth, W Renn. University of Tuebingen, Germany (Introduced by NP Quinn)

The authors studied the recovery of the hypothalamic-pituitary-adrenal (HPA) axis from inhibition by a short term intravenous high dose prednisolone therapy (1000 mg Prednisolone IV per day for five consecutive days) in 10 patients with relapsing remitting or progressive multiple sclerosis using the $\mathrm{CRH}$ (corticotrophin-releasing hormone) test.

Results indicate that there is a decrease in basal and stimulated ACTH and Cortisol output 24 hours after discontinuing Prednisolone. Eight of 10 patients showed a transient excessive response of increased basal ACTH to CRH with normalised Cortisol levels at 48 hours. This rebound phenomenon might be explained by an up-regulation of pituitary CRH-receptors during suppression. In the majority of patients the pattern of ACTH response was back to normal at 120 hours.

It is concluded that short term high dose intravenous steroid therapy in patients with multiple sclerosis is endocrinologically safe. Initial suppression of the HPA axis is reversed within 48 hours.

THE EPIDEMIOLOGY OF STEELE RICHARDSON OLSZEWSKI SYNDROME

SJWroe, RGWill, AJ Lees, Ipswich Hospital, Western General Hospital, Edinburgh and Institute of Neurology, London, UK

The Epidemiology of Steele Richardson Olszewski Syndrome (SRO) in the United Kingdom has been studied by examining death certificates for the seven year period, 1979-85. Death certificates classified under the Rubrick 333.0 (WHO 1987) were examined and standardised mortality ratios calculated for each region.

SRO was diagnosed in 128 patients (57 male, 71 female), median age at death 68 years. Shy Drager syndrome (80 cases) or Olivopontocerebellar degeneration (30 cases), were diagnosed less often. The number of cases of SRO varied from nil in Northern Ireland to 24 in South East Thames, the overall rate was $0 \cdot 33 / 1,000,000$, with the highest rate of $1 \cdot 8 / 1,000,000$ in South East Thames during the final three years of the study period. The number of cases of SRO decreased across the United Kingdom from South East to North West whereas no such pattern occurred for Shy Drager syndrome (average range $0 \cdot 21 / 1,000,000$ and highest regional rate $0 \cdot 6 / 1,000,000$ in East Anglia) where the distribution of diagnosed cases was more random.

There are large regional variations of a number of patients diagnosed as having SRO in the United Kingdom. Further studies should be aimed at establishing clear diagnostic criteria to allow syndrome recognition, prospective follow up with clinicopathological correlations, and community based prevalence studies which might give clues to aetiology. 